\title{
A time-division multiplexer-demultiplexer for duplex video recording and playback
}

\author{
A. RODNEY WELLENS \\ Department of Psychology, University of Miami, Coral Gables, Florida 33124 \\ and \\ RENE REVERT
Remote Sensing Laboratory, University of Miami, Coral Gables, Florida 33124
}

\begin{abstract}
A solid state circuit is described that allows two sources of video information to be multiplexed and recorded by one videotape machine. On playback, the same circuit is used to demultiplex the recorded video signal so that each of the original video channels may be independently viewed on separate television monitors.
\end{abstract}

Occasionally, the behavioral researcher has the need for simultaneously recording two separate channels of video information so that analyses can later be made regarding concurrent events. One popular method used for this kind of multichannel video recording makes use of a video mixer that divides and integrates two separate video inputs so as to produce a single splitscreen image (cf. Field, 1977; Shea \& Rosenfeld, 1976; Stephenson, Rutter, \& Dore, 1973). Video information received from one video source is split and assigned to a selected portion of the video field; the remaining part of the field displays a similarly fragmented image from the second video source. This technique is useful for some applications, but, since only part of the video field is available for recording and displaying each visual channel, it is undesirable when a high degree of picture resolution is needed.

A second recording method involves the yoking of two videotape machines for the synchronous recording and playback of two full-screen images. This requires the recording of a common digital time code on each videotape (e.g., Ekman, Friesen, \& Taussig, 1969). The time code is read on playback by a computer or synchronizing unit that locks the machines together through a series of servo mechanisms. While this solves the problem of restricted image size, the cost of the additional equipment needed is often high (some off-theshelf commercial systems cost in excess of $\$ 100,000$ ).

The device described here offers a relatively inexpensive alternative and has the advantage of allowing full-screen video recording and playback with a single videotape machine. This technique makes use of an externally provided synchronizing pulse to rapidly switch between video inputs such that every other video field recorded comes from a separate video source. On

Requests for reprints should be sent to A. Rodney Wellens, Department of Psychology, University of Miami, P.O. Box 248185, Coral Gables, Florida 33124. playback, the same switching mechanism outputs the alternate video fields to two display monitors, reestablishing two separate video channels. Switching occurs at a sufficiently rapid rate $(60 \mathrm{~Hz})$ to minimize problems associated with video flicker.

\section{TECHNICAL DESCRIPTION AND SYSTEM LOGIC}

Figure 1 shows the components of the multiplexing. demultiplexing device. A timing diagram is presented in Figure 2, which shows the transformation of an externally provided master synchronizing signal as it passes through the various integrated circuits contained in the device.

The video multiplexer-demultiplexer consists of four integrated circuits (ICs), seven resistors, one capacitor, one SPST switch, one SPDT switch, and seven UHF connectors. ${ }^{1}$ In either the multiplexing or the demultiplexing mode, an externally supplied master sync signal (see Figure 2a) is applied to the inverting input of comparator IC-1 via UHF connector A. IC-1 inverts the master sync input and acts as a level translator to raise the incoming $1-\mathrm{V}$ sync level to the required $5-\mathrm{V}$ level needed for IC-2. A 5-kohm potentiometer is used to set the reference voltage level at which comparator IC-1 will change its output state; the setting selected permits only the sync tips of the incoming signal to actuate the comparator. A 3-kohm potentiometer is used to trim any offset errors inherent in the individual comparator IC. The output from IC-1 (see Figure 2b) is applied to a retriggerable monostable multivibrator, IC-2, connected to act as a pulse-period differentiator. A $10-\mathrm{kohm}$ potentiometer adjusts the pulse width of IC-2. The potentiometer is set to approximately $3 \mathrm{kohm}$ so that the pulse width of the output signal is 80 microsec, which is greater than the horizontal line period of 63.5 microsec. The short time period occurring between horizontal sync pulses contained within the output 


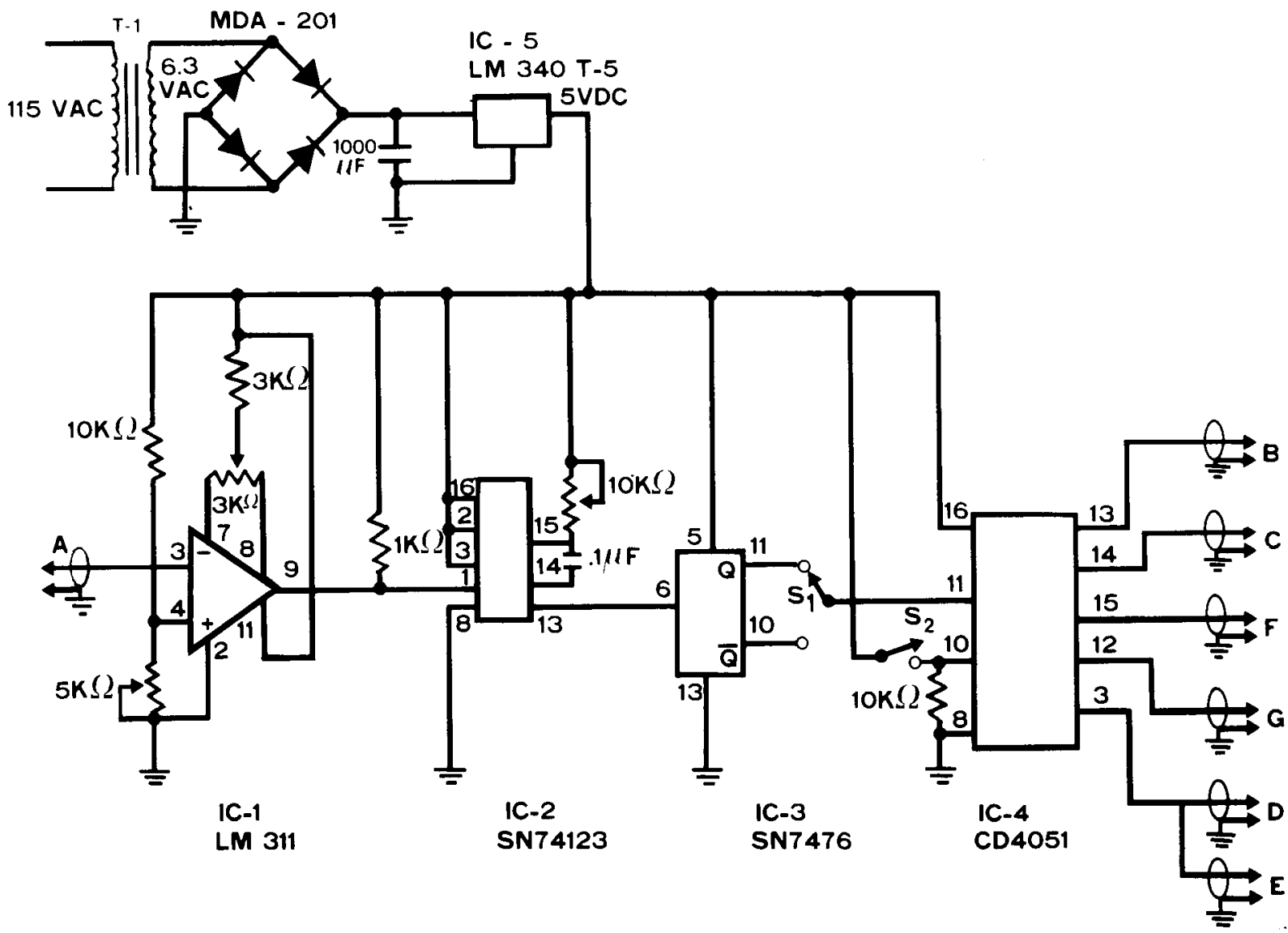

Figure 1. Schematic diagram of the video multiplexer-demultiplexer with its accompanying 5-V dc regulated power supply.

from IC-1 serves to constantly retrigger the multivibrator during the generation of any given field of video. During the relatively long interval representing vertical sync time (occurring at the end of each video field), the multivibrator returns to its resting state. The resulting output from IC-2 is thus devoid of horizontal sync information while retaining the $5-\mathrm{V}$ pulses that coincide with the vertical sync portion of the master sync signal (see Figure 2c). Finally, the output from IC-2 is applied to a flip-flop, IC-3, which toggles alternately, providing the alternating field pulse output depicted in Figure $2 \mathrm{~d}$.



Figure 2. Timing diagram depicting transformation of master sync pulse.
Within the multiplexing mode, the alternating field pulse output from IC-3 is applied to the least significant digital control input of an eight-channel analog multiplexer (IC-4). This is accomplished by placing $S_{2}$ in the open position. Given this particular input configuration, IC-4 alternately switches between two sources of video input provided through UHF connectors B and C. A time-multiplexed video signal is thereby produced and output via UHF connector D.

In the demultiplexing mode, the address of the digital control input to IC-4 is changed by closing $\mathrm{S}_{2}$; IC-4 now alternately switches between two video output devices via UHF connectors $F$ and $G$, effectively demultiplexing the video input provided through UHF connector $\mathrm{E}$. In order for switching to occur in phase with each frame of video received via connector $E$, the master sync signal applied to connector $\mathrm{A}$ must be externally gen-locked to the composite video signal being demultiplexed. (The gen-lock circuit serves to lock the externally generated master sync signal onto the sync pattern associated with the incoming video signal.)

After demultiplexing begins, one channel of video output is arbitrarily assigned to connector $\mathrm{F}$ and the other to G. If the user wishes to reverse the obtained output pattern, he may do so by switching $S_{1}$ from $\mathrm{Q}$ to $\overline{\mathrm{Q}}$, This manipulation simply reverses the on-off 
pattern of the digital control signal output from IC-3, causing IC-4 to reverse the video outputs to connectors $\mathrm{F}$ and $\mathrm{G}$.

\section{IMPLEMENTATION}

Figure 3 shows a typical wiring diagram for the video multiplexer-demultiplexer integrated into a CCTV system. The letters A-G in Figure 3 correspond to the UHF connectors with the same labels in Figure 1. The two input video sources are a pair of CCTV cameras (e.g., Panasonic Model WV-1150) with optional external sync controls. An external sync generator coordinates the vertical and horizontal scanning patterns of the two cameras. (Locking the two video sources together is necessary so that each generates a new field of video information at the moment switching occurs within the multiplexing circuit.) The same master sync signal triggers the switching cycle in the multiplexing circuit through connector $\mathbf{A}$.

The composite video outputs from Cameras 1 and 2 are alternately read by the multiplexing circuit through connectors $\mathrm{B}$ and $\mathrm{C}$. In the multiplexing mode, an alternating field composite video signal is available as output through connector $\mathrm{D}$. This signal may be used as a video input signal, containing both video and sync information, for any standard videotape recorder.

In the demultiplexing mode, the previously recorded alternating field composite video signal is inserted into the demultiplexing circuit through connector $\mathrm{E}$. The same signal is simultaneously fed to a gen-lock circuit within the external sync generator so that the sync output being fed to the demultiplexing circuit via connector $\mathrm{A}$ is concomitant with the composite video signal being demultiplexed. (Panasonic special effects generator Model WJ-545P has a built-in sync generator with an available gen-lock feature. Within the current example, the gen-lock circuit is engaged only during demultiplexing.) CCTV Monitors 1 and 2 receive demultiplexed video signals through connectors $F$ and $G$, respectively, on a skip-field basis. Since no sync information is available at connectors $F$ and $G$ between alternating fields, it is recommended that monitors have an external sync option (e.g., RCA Model TC1217). A continuous sync signal may be fed to each monitor via the gen-locked external sync generator in order to avoid rolling and/or picture breakup.

\section{APPLICATIONS}

The video multiplexer-demultiplexer has been used successfully to record the nonverbal behaviors of pairs of individuals engaged in social interactions conducted over two-way television (Wellens, 1978). The two CCTV cameras in Figure 3 are housed within the interactants' two-way television terminals. The availability on play. back of a full-screen close-up image of both participants in the dyadic exchange permits the subsequent analysis of sequential and concurrent looking patterns, as well as other synchronous nonverbal behavior (Bakeman \& Dabbs, 1976).

An alternative application involves focusing two CCTV cameras on a single subject to provide a multiperspective view of the same individual. This is useful

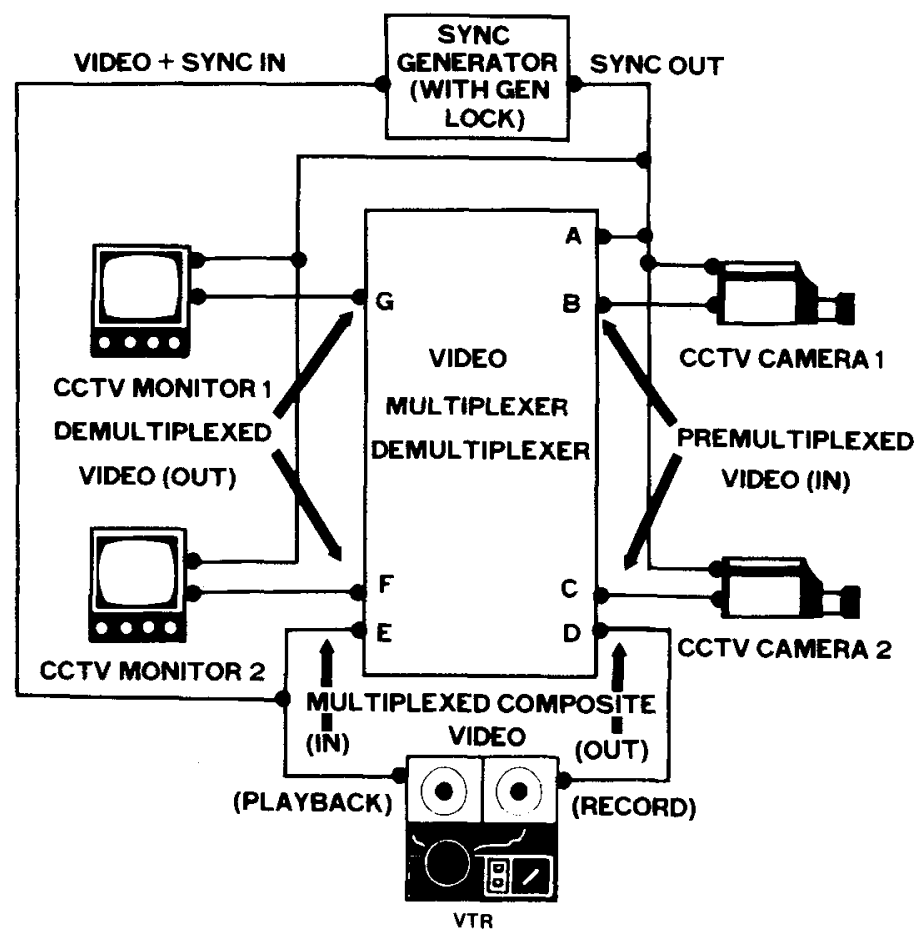

Figure 3. Typical wiring diagram for the video multiplexerdemultiplexer integrated into a CCTV system. 
when coding behaviors manifested along separate body axes. For example, interpersonal distance and forward body lean are better judged from a profile view (Mehrabian \& Ksionszky, 1972); eye movements are more accurately rated from a full-face perspective (Gibson \& Pick, 1963).

Another application involves recording behavioral responses to a visual event while simultaneously recording the event. If the target event is a live television broadcast or a videotape sequence, the signal can be piped directly into the video multiplexing circuit, replacing either CCTV Camera 1 or 2 in Figure 3. The remaining live CCTV camera is slaved to the sync signal accompanying the telecast or presecorded videotape. This is accomplished via the gen-lock circuit within the external sync generator used to trigger the multiplexing circuit.

\section{REFERENCES}

Bakeman, R., \& DabBs, J. Social interaction observed: Some approaches to the analysis of behavior streams. Personality and Social Psychology Bulletin, 1976, 2, 335-345.

Ekman, P., Friesen, W., \& Taussig, T. VID-R and SCAN: Tools and methods for the automated analysis of visual records. In G. Gerbner, O. Holsti, K. Krippendorff, W. Paisley, \&
P. Stone (Eds.), The analysis of communication content. New York: Wiley, 1969.

FIELD, T. M. Effects of early separation, interactive deficits, and experimental manipulations on infant-mother face-to-face interaction. Child Development, 1977, 48, 763-771.

Gibson, J., \& PICK, A. Perception of another's looking behavior. American Journal of Psychology, 1963, 76, 386-394.

Mehrabian, A., \& Ksionzky, S. Some determiners of social interaction. Sociometry, 1972, 35, 588-609.

Shea, M., \& Rosenfeld, H. Functional employment of nonverbal social reinforcers in dyadic learning. Journal of Personality and Social Psychology, 1976, 34, 228-239.

Stephenson, G., Rutter, D., \& Dore, S. Visual interaction and distance. British Journal of Psychology, 1973, 64, 251. 257.

WEllens, A. R. A device that provides an eye-to-eye video perspective for interactive television. Behavior Research Methods \& Instrumentation, 1978, 10, 25-26.

\section{NOTE}

1. For convenience, the upper portion of Figure 1 also contains a schematic for a $5-\mathrm{V}$ dc regulated power supply needed to operate the multiplexing-demultiplexing circuit. The power supply consists of a $115-\mathrm{V}$ to $6.3-\mathrm{V}$ transformer (T-1), a diode bridge (MDA-201), a capacitor, and a voltage regulator integrated circuit (IC-5).

(Received for publication February 26, 1979; revision accepted April 13,1979.) 